\title{
The Revelation of Cyber-Physical Internet (CPI)
}

\author{
Khalid A. Fakeeh, PhD \\ FCIT, King Abdullaziz University \\ Jeddah, Saudi Arabia
}

\begin{abstract}
When the Internet was conceived, the rationale was to interconnect Personal Computers to share advanced information or digital data everywhere. On the other hand, when implanted or embedded frameworks/networks were conceived, the motivation was to control system segments under real-time constrictions through detecting gadgets, ordinarily at little or small to medium scales. With the colossal development of the Information and Communication Technology (ICT), the propensity is to empower omnipresent persistent computing to control everything at whatever time and at a huge scale. This vision gave as of late ascent to the worldview of Cyber physical Systems (CPS). This article gives an idea of the Cyber-Physical Internet examine its outline prerequisites and present the constraints of the present systems/networks administration reflections to satisfy these necessities with a glimpse over real-time defies that must be considered in the outline Cyber-Physical Internet (CPI).
\end{abstract}

\section{Keywords}

IoT, CPS, Ubiquitous Computing

\section{INTRODUCTION}

The vision towards substantial scale distributed computing systems is presently advancing to other boondocks, where computation is no more decoupled from its surroundings. This sight originates from the need to coordinate outer physical information and procedures with calculations for purpose of pervasive and universal control of the encompassing milieu. On the other hand, it is ordinarily realized that this incorporation is not another idea as it has dependably been the situation with embedded systems. Truth be told, embedded computing systems are inherently subject to their surroundings where they are sent through detecting physical procedures or sensing physical processes. As computing turns out to be progressively incorporated into our surroundings, conventional embedded systems has discovered their cutoff points in fulfilling the new prerequisites of hugely arranged or networked embedded systems. Then again, the Internet has been giving an overall foundation to information sharing and data recovery. In any case, Internet applications have been driven by the need to trade consistent data everywhere on a large scale; in any case, the mapping between the physical environment and the legitimate data has not been considered in the configuration of those applications. Consequently, the Internet's joining with embedded systems is a critical development for empowering huge scale distributed computing systems that are firmly combined with their physical surroundings. From one perspective, an initial move towards this union has been put into practice by RadioFrequency identification (RFID) based frameworks or systems, which have empowered the idea of system of physical objects, generally known as Internet-of-Things (IoT) [I. T. Union]. In November 2005, the International Telecommunication Union (ITU) put forward an official statement in the World Summit on the Information Society that outlines the fundamental ideas of Internet of Things, related advances, difficulties and concerns, market opportunities, and so forth. Obviously, RFID has been considered as the key innovation for Internet-of-Things (IoT). Then again, the Wireless Sensor Networks (WSNs) worldview has risen as another different option for networks of physical events, which underpins the control and observing of physical wonders in nature through detecting or sensing. Some different choices considered the utilization of sensorbased cellular telephones for checking regular things through cell networks [C. Frank et al]. These methodologies fall into the idea of Cyber-Physical Systems (CPS), which are systems sent in huge geological territories and for the most part comprise of a monstrous number of distributed system devices firmly combined with their physical environs. Figure 1 shows the fundamental components of CPS
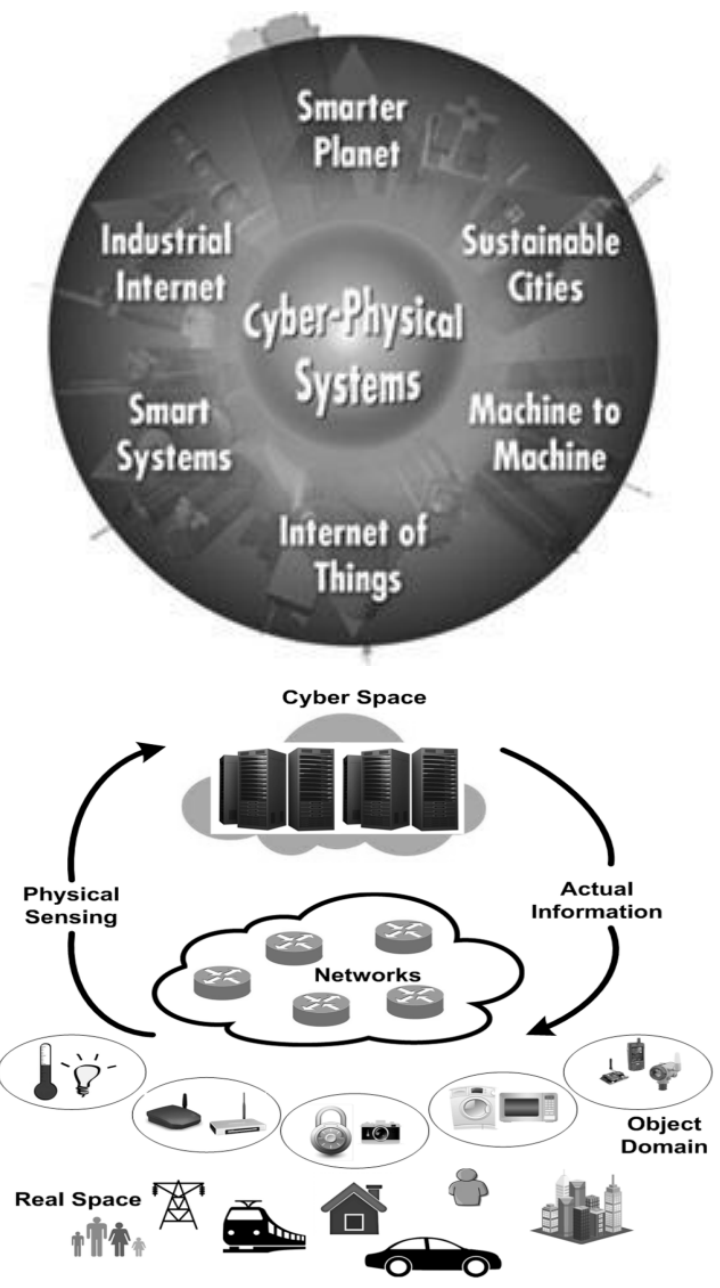

Fig 1: CPS main components 
The outskirts in the middle of CPS and Internet-of-Things (IoT) has not been obviously recognized subsequent to both ideas have been driven in parallel from two autonomous groups i.e. sensor networks and RFID, separately, in spite of the fact that they have dependably been firmly related. The history returns itself as this circumstance may be thought to be like the Internet's configuration driven by the TCP/IP group and Telecommunication systems/networks driven by International Telecommunication Union (ITU) in the mid eighties. Then again, with the development of the as of late discharged 6LoWPAN [G. Mulligan], the meeting in the middle of CPS and (IoT) turns into a genuine truth as it empowers to utilize the Internet as steady or accommodating framework/infrastructure to sensor systems, correspondingly to its incorporation with RFID frameworks/systems. The present status of these new developing CPS's review to the psyche the age simply going before the Internet's conception, when systems were scattered and private for the most part because of absence of principles. Likewise, CPS's are as of now scattered/distributed and private systems, each performs particular errands identified with nature where it works. The fundamental test in the configuration of CPS is the means by which to empower the interconnection and interoperability of all these scattered networked embedded systems into a solitary expansive scale arrange that fulfills every one of their prerequisites. There are various debilitations that upset the set-up of a brought together network for CPS's. Likewise, one critical inquiry is whether it would be better that the outline of CPS takes after a system joining or incorporated methodology, which comprises in coordinating heterogeneous systems together to shape a CPS, or a radical configuration approach as it was asserted in a few papers [CPS executive summary, 2008][ E. A. Lee][ Cyber-physical systems, 2006], which comprises in building CPS without any preparation.

\section{MODEL PREDICAMENT}

As of the ascent of Cyber Physical Systems, there were various calls to reexamine the computation foundations to cross other boondocks towards future CPS networks [CPS executive summary, 2008][ E. A. Lee][ Cyber-physical systems, 2006]. This is a truly charming enunciation to trigger new speculative investigation difficulties; in any case, in the rational sense, it may defy certified confinements. Regardless of the way that it is obvious that count perfect models must be changed in accordance with the new requirements that rise with the cutting's improvement edge cyber physical progressions, grasping a radical setup approach, as it might be understood from the research [ E. A. Lee][ Cyber-physical systems, 2006], is all in all not practical, at any rate in medium to short terms. In the midst of the modeling process of significant scale complex systems, it is continually fundamental that arranged models ensure the best trade off between their unmistakable requirements; in any case, when taking a look to history we can comprehend that bonafide or real systems that spread out into the business area not by any stretch of the creative ability fulfill the goals of the speculatively anticipated models. An immediate inquiry is that Will CPS's defy the similar doom? Genuinely, two general standard advances perfectly embed this conviction: Internet Protocol (IP) and IEEE 802.11 (starting now and into the foreseeable future, WiFi). These two standard protocols are by and large referred to not to some degree poor to the extent adequacy and Quality-of-Service (QoS). A couple patches have been proposed for IP, for instance, Integrated Services, Differentiated Services, et cetera and for WiFi e.g. IEEE 802.11e expansion to enhance their execution. Despite profitability and QoS do not have, these two protocols have been for the most part and quickly spreading ensuing to their release. Of course, other more refined protocols such ATM, X.25 or HyperLan have been created with more care to accomplish superior effectiveness and better QoS, however did not get an over the top measure of space in the business market. It gives the thought that there is constantly an opening between how new-fangled structures or systems are depended upon to work and how they function in veracity. The reason is that the market's vision accomplices is not the same as the vision of academic authorities, as the past couldn't think less about upgraded profitability, yet rather decrease a perfect time-to-market and cost of certifiable things. Along these lines, it can be successfully seen that, in sensible terms, the showing perspective is to quickly plan, complete and put-intobusiness segment direct courses of action that (i) just work, (ii) fulfill crucial essentials and (iii) can be altered to plug new functionalities or to upgrade their practices. Thusly, it gives the idea that reconsidering the present estimation foundations to manufacture endless scale Cyber Physical Systems is not in any case minded. Maybe, it gives the idea that it is more ordinary to take advantage from the legacy base to perform the generous scale CPS destinations. Thus in this way it is assume that examination attempts must focus on the system incorporation approach for enabling limitless scale Cyber Physical Systems, which is therefore imply as the CyberPhysical Internet (CPI). In this manner, interoperability is the key test to fabricate broad scale heterogeneous cyber physical frameworks/networks. Moreover, it is imperative to think about the specificities of Cyber Physical Systems in the process of integration of vacant frameworks/networks, particularly the method for the controlled data. One problem may thusly go up that what will be the inside or core protocol for the up and coming CPI. Beyond question, IP is the legacy protocol that will accept the key part later on CPI. IP has been deliberated for so long — since the sensor's origination network perspective - as being non flawless with the necessities of sensor-based systems. Regardless, this thought has been starting late come back to [A. Dunkels][K.Mayer et al][ J. W. Hui et al] and with the improvement of 6LoWPAN [G. Mulligan] [J. W. Hui et al] that embeds IPv6 on top of the IEEE 802.15.4 [The IEEE 802.15.4, 2006] as an alternate choice for ZigBee [ZigBee-Alliance, 2006] Network Layer. The test has been won by the IETF 6LoWPAN Working Group and 6LoWPAN transforms into a honest to goodness contender to ZigBee as it engages to reliably join the sensor framework world with the Internet, which ZigBee is not prepared to. In any case, if 6LoWPAN wins similarly as abnormal state of interoperability with existing frameworks/Networks, in spite of all that it needs to legitimize its efficiency in regards to energy capability and progressing confirmations. Surely, the blueprint destinations of 6LoWPAN are inclined to put more weights on interoperability and joining with the Internet rather than on the ordinary essentials of sensor frameworks or networks. Propelling the tradeoff between those design or model intentions remains an investigation defies.

\section{CPI NETWORKING GENERALIZATION}

Around there, the essentials are portrayed that need to well thought-out in the arranging's specific considerations of the Cyber-Physical Internet (CPI), which can by and large be seen as the tremendous scale complete network that interconnects a couple of heterogeneous CPS. A CPS is considered as a mix 
of a couple and differing networks that screen physical things and events, including RFID-based systems, WSNs, cell phones, et cetera. Future enormously networked embedded systems oblige new measures for achieving interoperability. Surely, inevitable broad scale CPS should not be expected as secluded and scattered structures, yet rather as a united system that reliably interconnects heterogeneous cyber physical parts. The test will be to amass an overall framework that interconnects all advanced physical devices and give $\mathrm{P} \& \mathrm{P}$ (plug \& Play) services to the end-customers in an absolutely clear way. It is in this way critical to reconsider the present networking reflections to ensure a general interoperability of cyber physical devices. This essential powers the setup and the change of new standardized protocols for CPS's. These protocols must be made while considering the world's properties, where the cyber physical systems will be sent. The IP protocol stack model and the WSN protocol stack model [L. F. Akyildiz et al] highlight essential hindrances that must be tended to in the diagram of the Cyber-Physical Internet (CPI). Frankly, the present protocols layers make a total reflection in transit of data to be arranged, in this way it is unrealistic to arrange protocols immovably consolidated with their outside environment. It is thusly essential to propose an increased protocol stack model for cyber physical systems that in like manner joins the properties of the physical circumstances. Figure 2 shows the probable reference expansion demonstrating for the Cyber-Physical Internet.

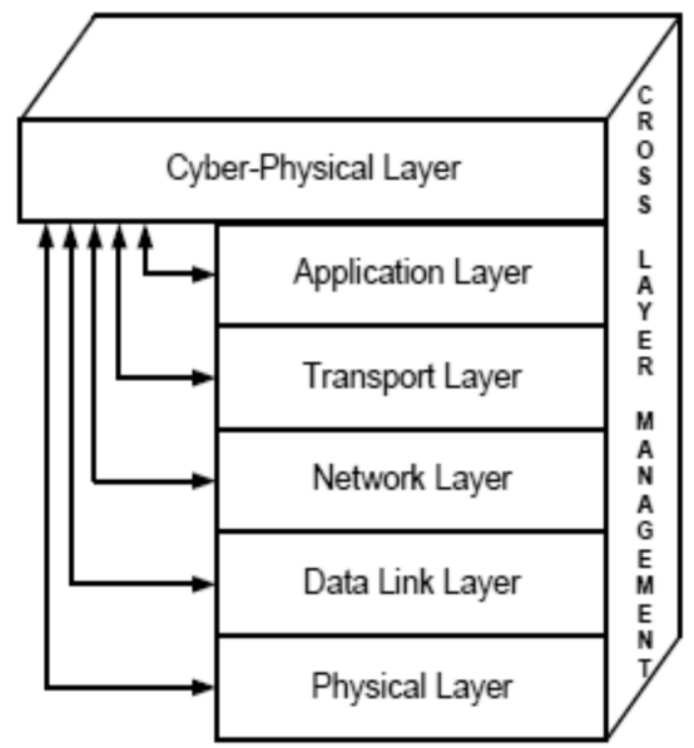

Fig 2: CPI Protocol Stack

The protocol stack basic making arrangements for CyberPhysical Internet must join an additional layer, the CyberPhysical Layer (CY-PHY layer), which gives an one of a kind delineation of the properties and nature of cyber physical data. This layer must bestow the protocols set to by and large identify with data in a united and sorted out way. On top, the Cyber-Physical Layer should offer services to lower layer protocols to reinforce a powerful cross-layer layout of the shrouded application and correspondence protocols. This infers that all protocols layers need to alter their behavior according to the information bestowed by the Cyber-Physical layer. For instance, in the setting of health monitoring, the information bestowed or granted by the body sensors will have a gigantic impact on the protocol's behavior suite expected for this application. Frankly, dependent upon the sort and the method for advanced physical data, a couple changes may be constrained in the protocol layers, to be particular:

(1) Physical Layer: The data from the CY-PHY layer can incite changing a couple of properties of the physical channel, for instance, the channel frequency band and the modification arrangement dependent upon the necessities of the cyber physical data. It can be conceived that some frequency bands can be owed for critical cyber physical traffic bringing into play solid regulation arrangements. Note that there have been unimaginable advances in radio advances with the design of cognitive radios and software radios, which offer better versatility to modifying radio properties to the customer necessities.

(2) Medium Access Layer: This layer generally yields diverse services and operational modes for higher-layer protocols, for instance, synchronized or unsynchronized modes; unmistakable levels of Quality-of-Service (QoS), energy management services etc. In CPS, the dynamic behavior of the milieu would out and out impact the operational behavior of the MAC layer, which must be adaptable to the Cyber Physical layer data. For example, the decision to change from synchronized operational mode to unsynchronized mode or the adaption duty cycle must be driven by the Cyber Physical layer (CY-PHY) layer. This can be expert by some present advances, for instance, the IEEE 802.15.4 protocol, which put forward the beacon-enabled mode "synchronized" and the non beacon-enabled mode "unsynchronized" in it MAC layer. This relationship between the MAC layer and CY-PHY layer is discriminating to engage a close loop control of the QoS based composed with the status of the checked surroundings.

(3) Network Layer: This layer bestows coordinating and data aggregation services. The cross-layer coordinated effort between the Network Layer and the CY-PHY Layer is vital to describe the adequate directing or routing systems and data all out segments. Case in point, the aggregation limits brought into play for processing temperature information would be absolutely extraordinary in connection to those employed for accelerometer or bio-medical unmistakable or sensory data. Besides, the assortment of routing methods or the parameters affecting a given routing protocol may depend on upon the data's route or its nature or moreover from the milieu type.

(4) Transport Layer: These protocols have not been comprehensively analyzed for CPS e.g. WSN's, embedded systems, despite the way that it is of an important criticalness to show unmistakable degrees of steadfastness concerning the end-to-end movement of data. This really proposes three conventional endeavors in this layer together with (a) tried and true transport, (b) flow control and (iii) congestion control methods. The Internet starting now gives the affiliation based TCP and connectionless UDP transport protocols for bestowing definite and best-effort services, independently. These heavyweight protocols are not suitable for the applications of CPS, which lift up the need to reconsider innovative transport protocols that adjust to the essentials and properties of Cyber Physical System. A bonafide test concerning transport protocols is to arrange tried and true transport protocols without the need to send back assertions to the source centers to go without choking out the network with the traffic control extension.

(5) Application Layer: This layer is accountable of taking care of data and uprooting profitable information with respect to the application destinations in Cyber Physical System. One 
essential test is to give standard appropriated sign planning algorithms/protocols for every potential Cyber Physical System applications. This will bolster the headway of Cyber Physical System applications and trim down a time to-market and outlay.

\section{PROSPECT PROGRESSING DEFIES}

Persistent (real-time) by and large powers bonafide troubles in the blueprint of CPS. Regardless, this issue must be caretotally dismembered and a thoughts' segment must be come back to. There are a couple promising investigation headings in the nonstop area. In what takes after, an example of prospective defies are given away:

(A) Network Protocols: From the networking point of view, ceaseless strengths a couple of troubles still open to explore. Flexible and distributed resource assignment in synchronized multi-hop sensor frameworks/networks, where resources must be adequately disseminated depending of the physical or logical framework/network changes, addresses one out of the ordinary investigation issue. In synchronized wireless sensors networks, it is typically more gainful to permit resources (information exchange limit/bandwidth, memory) to active sensor center points incorporated into fundamental errands. The usage of static allocation plans is clearly not beneficial for significantly dynamic and mobile systems as they don't acclimate to the system changes. Of course, the consolidated adaptable synchronization influences a considerable measure of estimation and correspondence/communication overheads, which can't for the most part work in resources, obliged wireless sensors networks, due to its complication and nonresponsiveness. We need to find new procedures for adaptively administering resources for synchronized and mobile multi-hop wireless sensors networks in a coursed, compelling, clear, or more all persistent approach.

(B) Operating Systems: It is indispensable that O.S supports real-time, in spite of the way that it incites additional arrangement multifaceted nature. The essential test is to perform a perfect concordance between a couple of basic segments needed by a Cyber Physical System O.S, comprising measured quality, intense hardware/software split, hardware abstraction, real time and energy viability [Cyber-physical systems, 2006]. The most by and large used O.S, TinyOS, identifies with a promising response for CPS as it addresses the lion's share of those essentials. In any case, the nonattendance of steady sponsorship addresses a bona fide obstacle in TinyOS for developing continuous protocols and synchronization frameworks [A. Cunha et al]. The nonappearance of preemption and prioritization in TinyOS is an essential incapacitation for giving obvious timing behavior at center level. In various cyber-physical applications, where timing impediments must be respected, unfaltering quality and consistent are all that highly coupled. It is thusly key to consider timing confirmations for building trustworthy systems. Genuinely, the nonattendance of real-time in TinyOS has kept the entry of standard-conforming IEEE 802.15.4 protocol stack for both open-ZB [A. Cunha et al] and TKN executions [A. Kopke et al] [J.-H. Hauer]. In [P. Pagano et al], the inventors showed that the behavior of the IEEE 802.15.4 execution has been much tried and true when realized over ERIKA [The e.r.i.k.a], a promising real-time O.S embedded devices.

(C) The Compositionality of Performance: The end-to-end delay examination in CPS is a mind boggling and engaging issue, particularly in light of their heterogeneity. Routine end- to-end delay examination in a general sense gets system level deferral from portion/component level delays, and this perspective has been known not inefficient. More refined strategies use association or composition theories to change a multi-part system into an one-fragment/component system, thusly lessening the versatile nature of the end-to-end examination [P. Jayachandran et al] [Delay composition algebra]. In [P. Jayachandran et al] [Delay composition algebra] the inventors showed a complete structure to purposely change passed on distributed systems into a single system, which is used to infer the end-to-end schedulability of the inventive system. Notwithstanding the way that these works identify with a pioneer responsibility towards execution compositionality in distributed real-time systems, their development model — in light of the schedulability examination theory - is to some degree limited to periodic/aperiodic streams and steady execution times i.e. most critical situation execution time, which is lacking insipid for exhibiting heterogenous CPS applications. On the other hand, Network Calculus formalism, which relies on upon more insipid development models (portrayed by their upper bound twists) [J.-Y. Le Boudec et al], gives join approaches to reducing the examination of a multi-hop system to a single hop system by choosing an indistinguishable organization twist for the whole system. The connection examination, on the other hand, has two impediments: (i) The indistinguishable service curve for a given stream depends on upon a parameter, whose change is extremely brain boggling [L. Lenzini et al], (ii) The examination relies on upon ratelatency service curves, which is inadequate insipid for modeling services in heterogeneous CPS applications. Consequently, there is a need to find adequate system diminishment methods that rely on upon general model considerations for addressing traffic and services, and that consider system heterogeneity.

(D) Amassing or Aggregation of Data: The at hand Internet protocol layers make a total consultation in transit of data to be arranged. Then again, various CPS applications are not roused by the data itself but instead they are genuinely enthused about unusual state request about the physical world. It is plausible for a customer to request that each sensor passes on its sensor scrutinizing and a short time later registers the result in light of every one of those sensor readings. Yet, such a technique makes a huge measure of data action something that (i) extends the time expected to get the request's delayed consequence and (ii) wastes energy of sensor nodes. Performing information taking care of inside the framework/network, for occasion allowing changes to similarly process drawing incoming packets before sending, can provoke basic redesigns nevertheless. This is routinely implied as data aggregation, content-based framework/network [T. Abdelzaher]. In spite of its checking, three basic issues stay for the usage of such a philosophy in CPI

i) The specification for Query Language: There is a need to portray a lingo in which customers can describe their inquiries and these request should be implanted into the framework/network. The gathering of WSN is at present using barely balanced variants of SQL. Regardless, these SQL varieties are not sufficiently expressive. Case in point, in a circumstance where we wanting to recognize whether a course is sans ice, we may wish that sensor center points or nodes perform sign get ready locally something that is difficult to perform profitably with SQL. One approach could be however that a sensor grants customers to present a device driver on 
that sensor center point/node and this device driver goes about as a virtual sensor; a virtual sensor performs a computation in perspective of physical sensors. Case in point, a virtual sensor may pass on a Boolean value "honest to goodness" if there is ice close to this sensor and "false" for the most part. By then, SQL may be brought into play to express inquiries considering the virtual sensors. Frankly, bolster for such virtual sensors are starting now open in a software package called Global Sensor Networks (GSN) [K. Aberer et al] anyway it is brought into play to be continue running on an entry interfacing with a remote sensor sort out rather than be continue running on sensor center points/nodes themselves.

ii) Query organizing and streamlining: The examination gathering of databases has conveyed a wide written work on inquiry get ready of SQL request. It regularly expect that the cost (for case time) of an inquiry should be minimized and the request masterminding/improvement attempts to find a strategy for executing the request such that the cost is minimized. These works expect that the cost is ruled by disks gets to or CPU get ready. Then again, for data gathering in CPS, it is expected the obliged furthest reaches of the (remote) correspondence channel to be the standard bottleneck and in this way request progression should try to minimize that outlay. This is non-minor in light of the way that (i) finding out about the wireless compose topology and hindrance association between centers/nodes are obliged remembering the final objective to attempt parallel transmissions and (ii) a couple of operations can be performed at amazing capability, (for instance, MIN, MAX) with a sorted out MAC protocol [B. Andersson et al]; the inquiry analyzer must be aware of that probable when enjoying decisions in how to reprieve down a query of customer into operations.

iii) Credibility and reliability of Data: Exactly when a customer poses a question he needs to verify that the sensor readings are substantial. Since data gathering grants controlling center points or nodes to adjust the data payload, common end-to-end encryption/acceptance frameworks don't work. Along these lines, ensuring data reliability must be the network irreplaceable part.

(E) Timing Assurance: The obtainment of deterministic ceaseless sureties in impulsive wireless ad-hoc and sensor networks is considered as a crude issue. In the written work, a huge bit of the papers overseeing deterministic protections expect that channels are sans bungle. While this assumption may be ideal for outstandingly incredible and exceptional cases, where remote/wireless associations or links are to a great degree relentless and of a magnificent, most of this present reality applications refute this suspicion, since the remote's majority associations/links are frequently arranged in the faint region, where links are significantly variable and volatile. The considered consistent sureties must be come back to, as it is needed to find innovative means to portray deterministic execution under channel unsteadiness. Ensuring that a wireless network deterministically gives a deferral/delay bound would not look good. One interesting depiction is to accomplice an assurance level with each guaranteed delay bound. The objective of the related assurance level is to gauge the helplessness on the guaranteed deferral or delay bound. The essential thought involves in handling as far as possible taking into account the amount of possible retransmissions, and to quantifiably center the number's assignment of retransmissions in a given channel.

\section{CONCULUSION}

The incorporation of physical procedures with the processing is old enough. The Cyber Physical Systems which are being utilized today are more befuddled and humble scale in size when appeared differently in relation to the expected or anticipated Cyber Physical Systems (CPS) unbounded. The immense networking of embedded computing gadgets or devices like sensors and actuators is beginning of the development. The foreseen change that CPS will give this development is the strategy for correspondence with the physical world as like the Internet which changed the way the overall public participates with each other. Framework/network need to organize the enrolling devices which are passed on geographically and physical segments and thus, framework/network accept an imperative part to enable striking or astounding exchanges between the physical world and people. In addition, it is clear that WSANs serve as a framework's spine in various CPS. The vision towards far reaching scale distributed systems is at this time progressing to be another topic for creative work, where estimation and environment are joined and not any more isolated. For this, there is a need to mastermind outside physical information and methods with computations for explanation behind spread and broad control of the including environment. Truly, embedded computing systems are basically reliant on their surroundings where they are presented through recognizing physical courses of strategy. The new essentials of colossally created embedded structures are adequately fulfilled by the customary embedded systems as preparing finds the opportunity to be persistently melded into our environment. Of course, the far reaching affiliation is being given by the Internet to enable the information sharing and recovery. Regardless, Internet applications have been controlled by the need to share the true blue data on the massive scale gave the mapping between the physical environment and the sensible data has not been considered in the diagram of those applications. As requirements be, the Internet's joining with embedded systems is a fundamental point of view for drawing in enormous scale passed on distributed computing that are firmly consolidated with their physical milieu [igi-global].

\section{REFERENCES}

[1] "P. Jayachandran et al", "A delay composition theorem for real-time pipelines," in ECRTS '07: Proceedings of the 19th Euromicro Conference on Real-Time Systems. Washington, DC, USA: IEEE Computer Society, 2007, pp. 29-38.

[2] "J.-Y. Le Boudec et al", Network Calculus: A Theory of Deterministic Queuing Systems for the Internet (Lecture Notes in Computer Science), 1st ed. Springer, August 2001.

[3] "C. Frank et al", "The sensor internet at work: Locating everyday items using mobile phones," Pervasive and Mobile Computing, vol. 4, no. 3, pp. 421-447, Jun. 2008.

[4] "G. Mulligan", "The 6LoWPAN architecture," in EmNets '07: Proceedings of the 4th workshop on Embedded networked sensors. New York, NY, USA: ACM, 2007, pp. 78-82.

[5] "A. Kopke et al", "IEEE 802.15.4 symbol rate timer for telosb," TKN Technical Report Series TKN-08-006, Telecommunication Networks Group, Technische Universitat Berlin, Tech. Rep., May 2008. 
[6] “A. Dunkels", "Full TCP/IP for 8-bit architectures," in MobiSys '03: Proceedings of the 1st international conference on Mobile systems, applications and services. New York, NY, USA: ACM, 2003, pp. 85-98.

[7] "K. Mayer et al", "IP-enabled wireless sensor networks and their integration into the internet," in InterSense '06: Proceedings of the first international conference on Integrated internet ad hoc and sensor networks. New York, NY, USA: ACM, 2006, p. 5.

[8] "J. W. Hui et al", "IP is dead, long live IP for wireless sensor networks," in SenSys '08: Proceedings of the 6th ACM conference on Embedded network sensor systems. New York, NY, USA: ACM, 2008, pp. 15-28.

[9] "L. F. Akyildiz et al", "A survey on sensor networks," Communications Magazine, IEEE, vol. 40, no. 8, pp. 102-114, 2002.

[10] "A. Cunha et al", "Zigbee over tinyos: Implementation and experimental challenges," in 8th Portuguese Conference on Automatic Control (CONTROLO2008), Invited Session on "Real-Time Communications: from theory to applications, 2008.

[11] "E. A. Lee", "Cyber physical systems: Design challenges," in International Symposium on Object/Component/Service-Oriented Real-Time Distributed Computing (ISORC), May 2008, invited Paper.

[12] "A. Cunha et al", "open-ZB: an opensource implementation of the IEEE 802.15.4/zigbee protocol stack on TinyOS," in The Fourth IEEE International Conference on Mobile Adhoc and Sensor Systems (MASS07), October 2007.

[13] "J.-H. Hauer", "TKN15.4: An IEEE 802.15.4 MAC implementation for TinyOS 2," TKN Technical Report Series TKN-08-003, Telecommunication Networks Group, Technical University Berlin, Tech. Rep., March, 2009.

[14] "P. Pagano et al," "ERIKA and OpenZB: an implementation for real-time wireless networking," in 24th ACM Symposium on Applied Computing (SAC 2009), Poster Session, March 2009.
[15] "T. Abdelzaher", "CPS position paper: Towards an architecture for distributed cyber-physical systems," in NFS workshop on Cyber-Physical Systems.

[16] "B. Andersson et al", "A scalable and efficient approach to obtain measurements in CAN-based control systems," IEEE Transactions on Industrial Informatics, vol. 4, 2008.

[17] "L. Lenzini et al", "End-to-end delay bounds in FIFOmultiplexing tandems," in ValueTools '07: Proceedings of the 2nd international conference on Performance evaluation methodologies and tools. ICST, Brussels, Belgium, Belgium: ICST (Institute for Computer Sciences, Social-Informatics and Telecommunications Engineering), 2007, pp. 1-10.

[18] "K. Aberer et al", "Infrastructure for data processing in large-scale interconnected sensor networks," in The 8th International Conference on Mobile Data Management (MDM'07), 2007.

[19] "Delay composition algebra: A reduction-based schedulability algebra for distributed real-time systems," in RTSS '08: Proceedings of the 2008 Real-Time Systems Symposium. Washington, DC, USA: IEEE Computer Society, 2008, pp. 259-269.

[20] http://www.igi-global.com/chapter/cyber-physicalinternet/121251

[21] "Cyber-physical systems executive summary," Tech. Rep., March 2008.

[22] "Cyber-physical systems - are computing foundations adequate?" in Position Paper for NSF Workshop On Cyber-Physical Systems: Research Motivation, Techniques and Roadmap, October 2006.

[23] The e.r.i.k.a. enterprise realtime operating system, http://www.evidence.eu.com/

[24] "The IEEE 802.15.4 standard specification," Tech. Rep., 2006.

[25] "ZigBee-Alliance", "The ZigBee standard specification,” Tech. Rep., December, 2006. 